\title{
SOME PATH PROPERTIES OF ITERATED BROWNIAN MOTION
}

\author{
KRZYSZTOF BuRDZY \\ University of Washington
}

1. Introduction and main results. Suppose that $X^{1}, X^{2}$ and $Y$ are independent standard Brownian motions starting from 0 and let

$$
X(t)= \begin{cases}X^{1}(t) & \text { if } t \geq 0 \\ X^{2}(-t) & \text { if } t<0\end{cases}
$$

We will consider the process $\{Z(t) \stackrel{\text { df }}{=} X(Y(t)), t \geq 0\}$ which we will call "iterated Brownian motion" or simply IBM. Funaki (1979) proved that a similar process is related to "squared Laplacian." Krylov (1960) and Hochberg (1978) considered finitely additive signed measures on the path space corresponding to squared Laplacian (there exists a genuine probabilistic approach, see, e.g., Mạdrecki and Rybaczuk (1992)). A paper of Vervaat (1985) contains a section on the composition of self-similar processes.

Supported in part by NSF grant DMS 91-00244 and AMS Centennial Research Fellowship . 
The present paper is devoted to studying path properties of IBM. We want to examine how the lack of independence of increments influences the results and estimates which are well understood in the Brownian case. This may be viewed as a prelude to a deeper study of the process.

First, however, we will address the following problem.

Problem 1. Given $\{Z(t), t \geq 0\}$, can one determine $\{X(t), t \geq 0\}$ and $\{Y(t), t \geq 0\} ?$

We start with a remark that there are many examples of functions $f, g, \widetilde{f}$ and $\widetilde{g}$ such that

$$
f(g(t))=\widetilde{f}(\widetilde{g}(t))
$$

for all $t \geq 0$ although $f$ and $\tilde{f}$ are not identically equal to each other and neither are $g$ and $\widetilde{g}$. Indeed, given any $f$ and $g$, identity (1) will be satisfied if we take $\widetilde{f}(t) \stackrel{\text { df }}{=} f(t / 2)$ and $\widetilde{g}(t) \stackrel{\text { df }}{=} 2 g(t)$. This simple observation does not imply the negative answer to Problem 1 because if $\{X(t), t \geq 0\}$ and $\{Y(t), t \geq 0\}$ are "typical" Brownian paths then $\{X(t / 2), t \geq$ $0\}$ and $\{2 Y(t), t \geq 0\}$ are not typical - they have the "wrong" quadratic variation.

Suppose that processes $X$ and $Y$ are defined on a probability space $\Omega$. We will write $X^{\omega}(t)$ and $Y^{\omega}(t)$ to indicate the dependence on $\omega \in \Omega$. 
Theorem 1. There is a set $N \subset \Omega$ of probability 0 such that if $\omega, \omega^{\prime} \in \Omega \backslash N$ and

$$
X^{\omega}\left(Y^{\omega}(t)\right)=X^{\omega^{\prime}}\left(Y^{\omega^{\prime}}(t)\right)
$$

for all $t \geq 0$ then either

$$
X^{\omega}(t)=X^{\omega^{\prime}}(t), t \in \mathbb{R}, \quad \text { and } \quad Y^{\omega}(t)=Y^{\omega^{\prime}}(t), t \geq 0,
$$

or

$$
X^{\omega}(t)=X^{\omega^{\prime}}(-t), t \in \mathbb{R}, \quad \text { and } \quad Y^{\omega}(t)=-Y^{\omega^{\prime}}(t), t \geq 0 \text {. }
$$

Problem 2. Given $\{X(X(t)), t \geq 0\}$, can one determine $\{X(t), t \geq 0\} ?$

Theorem 2. There is a set $N \subset \Omega$ of probability 0 such that if $\omega, \omega^{\prime} \in \Omega \backslash N$ and

$$
X^{\omega}\left(X^{\omega}(t)\right)=X^{\omega^{\prime}}\left(X^{\omega^{\prime}}(t)\right)
$$

for all $t \geq 0$ then $X^{\omega}(t)=X^{\omega^{\prime}}(t)$ for all $t \in \mathbb{R}$.

The following problem is analogous to (1). Do there exist continuous functions $f: \mathbb{R} \rightarrow \mathbb{R}$ and $\tilde{f}: \mathbb{R} \rightarrow \mathbb{R}$ such that $f(0)=\widetilde{f}(0)=0$ and

$$
f(f(t))=\tilde{f}(\tilde{f}(t))
$$

for all $t \geq 0$ ? 
Example 1. We will construct functions $f$ and $\widetilde{f}$ which satisfy (2) and "look like" Brownian paths. Choose any continuous function $f$ which satisfies the following conditions.

(i) $f(t)=f(-t)$ for all $t \geq 0$,

(ii) $f(t) \in[0,1]$ for all $t \in \mathbb{R}$,

(iii) $f(t)=f(1-t)$ for all $t \in[0,1]$,

(iv) $f(0)=0$ and $f(2)=1 / 2$,

(v) $f(t) \neq 1 / 2$ for some $t>2$,

(vi) $f$ is nowhere differentiable and the quadratic variation of $f$ over $[s, t]$ is equal to $t-s$ for all $0<s<t<\infty$.

Then let $\tilde{f}(t)=f(t)$ for $t \in[-2,2]$ and $\tilde{f}(t)=1-f(t)$ for $|t| \geq 2$. It is easy to see that there exist functions $f$ and $\tilde{f}$ satisfying all of the above conditions. It is elementary to check that functions $f$ and $\widetilde{f}$ satisfy (2).

The above example is not totally satisfactory because it hinges on the fact that the functions $f$ and $\tilde{f}$ are bounded and, therefore, they lack one of the simplest properties of Brownian paths. We give a proof of Theorem 2 which uses the same rather deep properties of Brownian paths which are utilized in the proof of Theorem 1. One may ask whether Theorem 2 may be proved using only simple facts about Brownian trajectories.

Problem 3. Suppose that $X$ is a Brownian sheet and $Y$ is a two-dimensional Brownian motion starting from $(2,2)$ and 
stopped at the hitting time $T$ of the circle $\left\{z \in \mathbb{R}^{2}: \mid z-\right.$ $(2,2) \mid=1\}$. Let $Z(t)=X(Y(t))$. Given $\{Z(t), t \geq 0\}$ and $Y(T)$, can one determine $\{Y(t), t \geq 0\}$ ?

It is quite obvious that $Z$ cannot determine $X$ as $Y$ visits only a compact subset of the plane. We do not know the answer to Problem 3.

Now we turn to the Law of Iterated Logarithm. A standard proof of LIL for Brownian motion (Karatzas and Shreve (1988) 2.9.23) uses the independence of Brownian increments. Since the increments of IBM are not independent, one may wonder whether a version of the LIL holds for IBM and how difficult it is to prove. Let us start with some simple observations. By the usual LIL, a.s. for every $\varepsilon>0$ there exists $t_{0}=t_{0}(\varepsilon)>0$ such that

$$
|X(t)| \leq(1+\varepsilon) \sqrt{2 t \log \log (1 / t)}
$$

and

$$
|Y(t)| \leq(1+\varepsilon) \sqrt{2 t \log \log (1 / t)}
$$

provided $|t|<t_{0}$. Hence for small $t>0$,

$$
\begin{aligned}
|Z(t)|= & |X(Y(t))| \\
\leq & (1+\varepsilon) \sqrt{2(1+\varepsilon) \sqrt{2 t \log \log (1 / t)}} \times \\
& \quad \times \sqrt{\log \log (1 /(1+\varepsilon) \sqrt{2 t \log \log (1 / t)})} \\
\leq & (1+\varepsilon)^{2} 2^{3 / 4} t^{1 / 4}(\log \log (1 / t))^{3 / 4} .
\end{aligned}
$$


Since $\varepsilon>0$ is arbitrary, we obtain

$$
\limsup _{t \rightarrow 0} \frac{|Z(t)|}{t^{1 / 4}(\log \log (1 / t))^{3 / 4}} \leq 2^{3 / 4} \quad \text { a.s. }
$$

Theorem 3 below shows that this result is not sharp. The reason is that in its derivation we have assumed the "worst case" scenario in which some unusually large increments of $X$ match those of $Y$. Thus it is no surprise that the constant $2^{3 / 4}$ on the right hand side of the last formula is replaced with a smaller constant $2^{5 / 4} / 3^{3 / 4}$ in Theorem 3 .

The lower bound in the proof of LIL for Brownian motion uses the part of the Borel-Cantelli Lemma which requires independent events. It is perhaps worth noting that despite the lack of independence of increments of $Z$, the proof of LIL for IBM is not much harder than that for Brownian motion. This may serve as an encouragement to further study of IBM.

Theorem 3. With probability 1,

$$
\limsup _{t \rightarrow 0} \frac{Z(t)}{t^{1 / 4}(\log \log (1 / t))^{3 / 4}}=\frac{2^{5 / 4}}{3^{3 / 4}}
$$

Theorem 3 and standard arguments easily imply that

$$
\limsup _{h \downarrow 0} \frac{Z(t+h)-Z(t)}{h^{1 / 4}(\log \log (1 / h))^{3 / 4}}=\frac{2^{5 / 4}}{3^{3 / 4}} \quad \text { a.s. }
$$

for every $t>0$. 
We are grateful to Carl Mueller and Terry McConnell for supplying references. We would also like to thank Rich Bass, Davar Khoshnevisan, Andrzej Mạdrecki, Ron Pyke, Paul Tseng and Wim Vervaat for the most useful discussions. We gratefully acknowledge that the idea of Example 1 is due to an anonymous person who kindly responded to our inquiry.

\section{Proofs.}

Proof of Theorem 1. The set $N \subset \Omega$ will contain several families of "exceptional" paths. First of all, we will choose $N$ so that for all $\omega \in \Omega \backslash N$ the functions $t \rightarrow X^{\omega}(t)$ and $t \rightarrow Y^{\omega}(t)$ are continuous, non-constant and non-monotone on every interval and satisfy $X^{\omega}(0)=Y^{\omega}(0)=0$. Hence, the local maxima of $X^{\omega}$ are dense on the real line. We may also suppose that if $X^{\omega}$ attains local maxima at $t_{1}$ and $t_{2}$ then $X\left(t_{1}\right) \neq X\left(t_{2}\right)$ (in other words, all local maxima are distinct). Moreover, we can assume that for $\omega \in \Omega \backslash N$

$$
\limsup _{t \rightarrow \infty} Y^{\omega}(t)=-\liminf _{t \rightarrow \infty} Y^{\omega}(t)=\infty
$$

Suppose that $-\infty<a<b<\infty$ and let $M=\max _{t \in[a, b]} Y(t)$. Then with probability $1, X(M)$ is not a maximum of $X$ over any interval $[M-\varepsilon, M]$ or $[M, M+\varepsilon]$ for any $\varepsilon>0$. The same is true if we let $M=\min _{t \in[a, b]} Y(t)$. By taking the union over all rational pairs $(a, b)$ we may choose 
$N$ so that for $\omega \in \Omega \backslash N$ and every local extremum $M$ of $Y^{\omega}$, $X^{\omega}(M)$ is not a maximum of $X^{\omega}$ over any interval $[M-\varepsilon, M]$ or $[M, M+\varepsilon]$ for any $\varepsilon>0$. Let us call this property $(P)$. This completes our description of $N$ for now. We will throw in some more sets into $N$ later.

We will argue that if $M$ is a local maximum of $Z$ then it is also a local maximum of $X$. Suppose that $Z(t)=X(Y(t))=$ $M$ is a local maximum of $Z$ and let $s=Y(t)$. Assume that $M=X(s)$ is not a local maximum for $X$. Then for every $\varepsilon>0$ the process $X$ takes a value greater than $M$ in $[s-\varepsilon, s]$ or in $[s, s+\varepsilon]$ and without loss of generality we may assume that for every $\varepsilon>0$ we have $X(v)>M$ for some $v \in[s, s+\varepsilon]$. This and the fact that $M=Z(t)$ is a local maximum for $Z$ imply that there is some $\delta>0$ such that $Y(u) \leq s$ for all $u \in[t-\delta, t+\delta]$. In other words, $s=Y(t)$ is a local maximum for $Y$. Moreover, we must have $X(v) \leq M$ for all $v \in\left[s-\delta_{1}, s\right]$ for some $\delta_{1}>0$. Hence, $M$ is the maximum of $X$ over $\left[s-\delta_{1}, s\right]$. This and the fact that $s=Y(t)$ is a local maximum for $Y$ contradict property $(P)$. We conclude that if $M$ is a local maximum of $Z$ then it must be also a local maximum of $X$.

Vice versa, if $M$ is a local maximum of $X$ then it is also a local maximum of $Z$. Suppose that $M=X(t)$ is the maximum of $X$ over $(t-\varepsilon, t+\varepsilon)$ for some $\varepsilon>0$. Find $s$ and $\delta>0$ such that $Y(s)=t$ and $Y(u) \in(t-\varepsilon, t+\varepsilon)$ for $u \in(s-\delta, s+\delta)$. Then clearly $M$ is the maximum of $Z$ over $(s-\delta, s+\delta)$. 
Let us list some elementary properties of IBM $Z(t)$ which hold for all $\omega \in \Omega \backslash N$. We have $Z(0)=0$, the function $t \rightarrow Z(t)$ is continuous, non-constant and non-monotone on every interval. Hence, the set of times when $Z$ attains a local maximum is dense in $[0, \infty)$.

We remark parenthetically that if $M=Z(t)$ is a local maximum of $Z$ then for each $\varepsilon>0$ the interval $[t-\varepsilon, t+\varepsilon]$ contains uncountably many $s$ such that $Z(s)=M$. We skip an easy proof as we do not need this fact in the sequel. We have to remember though that two local maxima of $Z$ attained at different points may have the same value.

Let $\left\{M_{1}, M_{2}, \ldots\right\}$ be the sequence of the values of all local maxima of $Z$ ordered in an arbitrary way. We do not place the same value more than once in the sequence. In other words, $M_{j} \neq M_{k}$ if $j \neq k$. For $j \geq 1$, let $s_{j}$ be such that $X$ attains the local maximum $M_{j}$ at $s_{j}$. Recall that $s_{j}$ is uniquely defined. The local maxima $M_{1}, M_{2}$ and $M_{3}$ come in a certain order, say, $s_{1}<s_{3}<s_{2}$. We may gain some knowledge about the order in which they come by examining the path of $Z$ as follows. There exist $t_{1}<t_{3}<t_{2}$ such that $Y\left(t_{j}\right)=s_{j}$ for $j=1,2,3$ and $Y(t) \in\left(s_{1}, s_{2}\right)$ for all $t \in\left(t_{1}, t_{2}\right)$. It follows easily that $Z$ attains the local maximum $M_{j}$ at $t_{j}$ for $j=1,2,3$, and, moreover, if $Z$ attains a local maximum at $t \in\left(t_{1}, t_{3}\right)$ then this maximum cannot be equal to $M_{2}$. Likewise, any local maximum of $Z$ between $t_{3}$ and $t_{2}$ cannot be equal to $M_{1}$. It 
is easy to see that $Z$ may have the last two properties only if $s_{3}$ is between $s_{1}$ and $s_{2}$ (although the precise order may be $s_{1}<s_{3}<s_{2}$ or $\left.s_{2}<s_{3}<s_{1}\right)$. Our argument can be repeated for any triplet $\left(M_{j_{1}}, M_{j_{2}}, M_{j_{3}}\right)$ of local maxima of $X$.

We will build a function $\{\widetilde{V}(t), t \in(-\infty, \infty)\}$ and later "time-change" $\widetilde{V}$ to obtain $\{X(t), t \in(-\infty, \infty)\}$. First we will inductively define a function $V(u)$ for certain values of $u$. We start with $V(1) \stackrel{\text { df }}{=} M_{1}$ and $V(2) \stackrel{\text { df }}{=} M_{2}$. Suppose that the function $V$ is defined at points $u_{j}, 1 \leq j \leq k$, and $V\left(u_{j}\right)=M_{j}$ for $j \leq k$. Now we use the argument from the previous paragraph to determine for each triplet $\left(M_{j_{1}}, M_{j_{2}}, M_{j_{3}}\right), j_{1}, j_{2}, j_{3} \leq k+1$, of local maxima which maximum comes between the other two on the path of $X$. The set $A_{k} \stackrel{\text { df }}{=} \mathbb{R} \backslash \bigcup_{j \leq k}\left\{u_{j}\right\}$ consists of a finite number of intervals and we will choose $u_{k+1}$ in one of the intervals (say, $I$ ) so that for each triplet $\left(u_{j_{1}}, u_{j_{2}}, u_{j_{3}}\right)$, $j_{1}, j_{2}, j_{3} \leq k+1$, the point $u_{j_{1}}$ is between $u_{j_{2}}$ and $u_{j_{3}}$ if and only if $s_{j_{1}}$ is between $s_{j_{2}}$ and $s_{j_{3}}$ on the path of $X$. It is easy to see that such a choice of $I$ is possible and unique. To be more specific, if the endpoints of $I$ are $u_{j_{1}}$ and $u_{j_{2}}$ then we let $u_{k+1}=\left(u_{j_{1}}+u_{j_{2}}\right) / 2$. If the endpoints of $I$ are $u_{j_{1}}$ and $\infty$ then $u_{k+1}=u_{j_{1}}+1$ and finally, if the endpoints are $-\infty$ and $u_{j_{1}}$ then $u_{k+1}=u_{j_{1}}-1$. Then we let $V\left(u_{k+1}\right)=M_{k+1}$.

We will show that the function $V(u)$ is defined for a set of $u$ 's which is dense in $\mathbb{R}$. If there is $\widetilde{u}<\infty$ such that our procedure does not define $V(u)$ for $u>\widetilde{u}$ then there must exist 
the greatest or the smallest time when the Brownian motion $X$ attains a local maximum - this is impossible. For the same reason there is no finite lower bound for the set of $u$ 's for which $V$ is defined. Suppose that there exists an open interval $\left(v_{1}, v_{2}\right)$ such that $V(u)$ is not defined for any $u \in\left(v_{1}, v_{2}\right)$. We may suppose that $\left(v_{1}, v_{2}\right)$ is a maximal interval with this property and we see that because of the way we choose $u_{j}$ 's, we must have $v_{1}=u_{j_{1}}$ and $v_{2}=u_{j_{2}}$ for some $j_{1}$ and $j_{2}$. This implies that there is no maximum on the path of $X$ between $s_{j_{1}}$ and $s_{j_{2}}$. This cannot happen and our assertion is proved.

Next we will show that $V$ can be continuously extended to $\mathbb{R}$. Recall that $X\left(s_{j}\right)=V\left(u_{j}\right)=M_{j}$. Let us define a function $\lambda$ on $\bigcup_{j}\left\{s_{j}\right\}$ by $\lambda\left(s_{j}\right) \stackrel{\text { df }}{=} u_{j}$. It follows from our construction of $V$ that the function $\lambda$ is strictly monotone. Moreover it cannot have jumps because then $\bigcup_{j}\left\{u_{j}\right\}$ could not be dense in $\mathbb{R}$. Hence, $\lambda$ and $\lambda^{-1}$ are well-defined, strictly monotone and continuous. Let $\widetilde{V}(u) \stackrel{\text { df }}{=} X\left(\lambda^{-1}(u)\right)$ for all real $u$. This function is a.s. continuous since it is a composition of two continuous functions. The functions $V$ and $\widetilde{V}$ agree on a dense subset of the real line, namely on $\bigcup_{j}\left\{u_{j}\right\}$. We conclude that $\widetilde{V}$ is a (necessarily unique) continuous extension of $V$ to the whole real line.

We have proved that $Z^{\omega}$ uniquely determines a continuous function $\widetilde{V}^{\omega}$ which is a (random) time-change of $X^{\omega}$ by a strictly monotone and continuous function $\lambda_{\omega}^{-1}$. Suppose that 
there exist $\omega$ and $\omega^{\prime}$ such that

$$
\widetilde{V}^{\omega}(u)=X^{\omega}\left(\lambda_{\omega}^{-1}(u)\right)=X^{\omega^{\prime}}\left(\lambda_{\omega^{\prime}}^{-1}(u)\right)
$$

for all $u$. Then there exists a strictly monotone and continuous function $\gamma=\gamma_{\omega, \omega^{\prime}}$ such that $X^{\omega}(t)=X^{\omega^{\prime}}(\gamma(t))$ for all t. Theorem 1.5.8 of Karatzas and Shreve (1988) easily implies the following two facts. First, with probability 1, for all rational $s$ and $t$, the quadratic variation of $X$ over $[s, t]$ is equal to $t-s$ (we add the trajectories which do not have this property to the set $N$ ). Second, for all rational $s$ and $t$, the quadratic variation of $X^{\omega}$ over $[s, t]$ is equal to the quadratic variation of $X^{\omega^{\prime}}$ over $[\gamma(s), \gamma(t)]$ assuming that $\omega, \omega^{\prime} \notin N$ (note that $\gamma$ maps a sequence of partitions of $[s, t]$ with the mesh going to 0 onto a sequence of partitions of $[\gamma(s), \gamma(t)]$ with the mesh going to 0$)$. The last two facts imply that $\gamma$ is a linear function with the slope equal to 1 or -1 . It follows that $\lambda^{-1}$ is unique up to an additive constant and up to the multiplication by -1 . We can find a sequence of local maxima $\left\{K_{j}\right\}_{j \geq 0}$ of $Z$ such that $Z\left(t_{j}\right)=K_{j}$ and $t_{j} \rightarrow 0$ as $j \rightarrow \infty$. Let $s_{j}$ be such that $\widetilde{V}\left(s_{j}\right)=K_{j}$ and $\widetilde{V}$ attains a local maximum at $s_{j}$. The $s_{j}$ 's are uniquely determined and they must converge to a point which corresponds to the origin on the path of $X$. More precisely, $s_{j}$ 's converge to $s$ such that $\lambda^{-1}(s)=0$. This determines $\lambda^{-1}$ up to the multiplication by -1 . Hence we either have $X^{\omega}(t)=X^{\omega^{\prime}}(t)$ for all $t \in \mathbb{R}$ or $X^{\omega}(t)=X^{\omega^{\prime}}(-t)$ for all 
$t \in \mathbb{R}$. The part of Theorem 1 concerned with the path of $X$ is proved.

Suppose that the paths of $Z$ and $X$ are given. If $Z$ attains a local maximum $M$ at $t$ then there exists a unique $s$ such that $X(s)=M$ and $X$ attains a local maximum at $s$. We must have $Y(t)=s$. Since the set of points where $Z$ attains a local maximum is dense on the real line, the path of $Y$ is determined on a dense set and, by continuity, on the whole real line.

Proof of Theorem 2. First we prove that the paths of $X$ have a property analogous to property $(P)$ introduced in the proof of Theorem 1. Suppose that $-\infty<a<b<c<d<\infty$ and let $M=\max _{t \in[a, b]} X(t)$. Given the values of $X(b)$ and $X(c)$, the processes $\{X(t), t \in[a, b]\}$ and $\{X(t), t \in[c, d]\}$ are independent. Hence, by conditioning on $X(b)$ and $X(c)$, one can easily prove that with probability $1, X(M)$ is not a maximum of $X$ over any interval $[M-\varepsilon, M] \cap[c, d]$ or $[M, M+$ $\varepsilon] \cap[c, d]$ for any $\varepsilon>0$. The same is true if we let $M=$ $\min _{t \in[a, b]} X(t)$. By taking the union over all rational numbers $a<b<c<d$ and $c<d<a<b$ we see that with probability 1 , if $M$ is a local extremum of $X$ and $X(M) \neq M$ then $X(M)$ is not a maximum of $X$ over any interval $[M-\varepsilon, M]$ or $[M, M+$ $\varepsilon]$ for any $\varepsilon>0$. For any fixed interval $[a, b]$ with rational endpoints, the maximum of $X$ over $[a, b]$ is not attained at a time $M$ such that $X(M)=M$, with probability 1. By taking 
the union over all such intervals, we see that with probability $1, X(M) \neq M$ for every local maximum of $X$. We conclude that almost all trajectories of $X$ have the property analogous to $(P)$.

Now we can follow the proof of Theorem 1 and arrive at the conclusion that for $\omega, \omega^{\prime} \in \Omega \backslash N$ the condition

$$
X^{\omega}\left(X^{\omega}(t)\right)=X^{\omega^{\prime}}\left(X^{\omega^{\prime}}(t)\right) \quad \text { for } \quad t \geq 0
$$

implies that either

$$
X^{\omega}(t)=X^{\omega^{\prime}}(t), t \in \mathbb{R}
$$

or

$$
X^{\omega}(t)=X^{\omega^{\prime}}(-t), t \in \mathbb{R}, \quad \text { and } \quad X^{\omega}(t)=-X^{\omega^{\prime}}(t), t \geq 0
$$

In the latter case we would have $X^{\omega}(t)=-X^{\omega}(-t)$ for $t \geq 0$. Such paths form an event of probability 0 .

Lemma 1. There exists $c_{1}<\infty$ such that for all $t, a>0$

$$
P\left(\sup _{0 \leq s \leq t}|Z(s)|>a\right) \leq c_{1} \exp \left(-3 \cdot 2^{-5 / 3} a^{4 / 3} t^{-1 / 3}\right) .
$$

Proof. Recall that for $a>0$ (cf. Karatzas and Shreve (1988)

(3) $\int_{y}^{\infty} \frac{1}{\sqrt{2 \pi t}} \exp \left(-x^{2} / 2 t\right) d x \leq \frac{1}{y} \sqrt{\frac{t}{2 \pi}} \exp \left(-y^{2} / 2 t\right)$. 
We derive two related estimates.

$$
\begin{aligned}
\int_{b}^{\infty} \sqrt{\frac{x}{t}} \exp \left(-x^{2} / 2 t\right) d x & \leq \int_{b}^{\infty} \sqrt{\frac{x}{t}} \sqrt{\frac{t}{b}} \sqrt{\frac{x}{t}} \exp \left(-x^{2} / 2 t\right) d x \\
& =-\left.\sqrt{\frac{t}{b}} \exp \left(-x^{2} / 2 t\right)\right|_{x=b} ^{x=\infty} \\
& =\sqrt{\frac{t}{b}} \exp \left(-b^{2} / 2 t\right) . \\
\int_{0}^{b} \sqrt{y} \exp \left(-a^{2} / 2 y\right) d y & \leq \int_{0}^{b} \sqrt{b} \frac{2}{a^{2}} \frac{a^{2}}{2} \frac{b^{2}}{y^{2}} \exp \left(-a^{2} / 2 y\right) d y \\
& =\left.\sqrt{b} \frac{2}{a^{2}} b^{2} \exp \left(-a^{2} / 2 y\right)\right|_{y=0} ^{y=b} \\
& =2 b^{5 / 2} a^{-2} \exp \left(-a^{2} / 2 b\right) .
\end{aligned}
$$

It is known (see Karatzas and Shreve (1988) 2.8.2) that the density of the random variable $\sup _{0 \leq s \leq t} Y(s)$ is equal to

$$
\sqrt{2 / \pi t} \exp \left(-y^{2} / 2 t\right)
$$

for $y>0$. It follows that the density of $\sup _{0 \leq s \leq t}|Y(s)|$ is bounded by

$$
2 \sqrt{2 / \pi t} \exp \left(-y^{2} / 2 t\right)
$$

for $y>0$. A similar formula holds for the density of the supremum of $X$. We may combine it with (3) to obtain

$$
\begin{aligned}
P\left(\sup _{0 \leq s \leq y} X(s)>a\right) & =\int_{a}^{\infty} \sqrt{2 / \pi y} \exp \left(-z^{2} / 2 y\right) d z \\
& \leq(1 / a) \sqrt{2 y / \pi} \exp \left(-a^{2} / 2 y\right)
\end{aligned}
$$


Hence, by symmetry,

$$
P\left(\sup _{-y \leq s \leq y}|X(s)|>a\right) \leq(4 / a) \sqrt{2 y / \pi} \exp \left(-a^{2} / 2 y\right) .
$$

If $\sup _{0 \leq s \leq t}|Y(s)|=y$ and $\sup _{0 \leq s \leq t}|Z(s)|>a$ then $\sup _{-y \leq s \leq y}|X(s)|>a$. Thus, by conditioning on the value of $\sup _{0 \leq s \leq t}|Y(s)|$ and using (6) and (7) we obtain

$$
\begin{aligned}
& P\left(\sup _{0 \leq s \leq t}|Z(s)|>a\right) \\
& \quad \leq \int_{0}^{\infty}(4 / a) \sqrt{2 y / \pi} \exp \left(-a^{2} / 2 y\right) 2 \sqrt{2 / \pi t} \exp \left(-y^{2} / 2 t\right) d y
\end{aligned}
$$

$$
=\frac{16}{\pi a \sqrt{t}} \int_{0}^{\infty} \sqrt{y} \exp \left(-a^{2} / 2 y-y^{2} / 2 t\right) d y .
$$

We will split the interval of integration in the last formula into three subintervals and estimate the integral for each one of them. It is elementary to check that the maximum of the function

$$
y \rightarrow \exp \left(-a^{2} / 2 y-y^{2} / 2 t\right)
$$

on the interval $[0, \infty)$ is attained at $u \stackrel{\mathrm{df}}{=}\left(t a^{2} / 2\right)^{1 / 3}$ and so

$$
\begin{aligned}
\exp \left(-a^{2} / 2 y-y^{2} / 2 t\right) & \leq \exp \left(-a^{2} / 2 u-u^{2} / 2 t\right) \\
& =\exp \left(-3 \cdot 2^{-5 / 3} a^{4 / 3} t^{-1 / 3}\right)
\end{aligned}
$$


for all $y>0$. This implies that

$$
\begin{aligned}
& \frac{16}{\pi a \sqrt{t}} \int_{2 u / 3}^{\sqrt{3} u} \sqrt{y} \exp \left(-a^{2} / 2 y-y^{2} / 2 t\right) d y \\
& \leq \frac{16}{\pi a \sqrt{t}} \sqrt{\sqrt{3} u} \exp \left(-a^{2} / 2 u-u^{2} / 2 t\right) u(\sqrt{3}-2 / 3) \\
& =c_{2} \frac{u^{3 / 2}}{a \sqrt{t}} \exp \left(-3 \cdot 2^{-5 / 3} a^{4 / 3} t^{-1 / 3}\right) \\
& =c_{2} \frac{\left(t a^{2} / 2\right)^{1 / 2}}{a \sqrt{t}} \exp \left(-3 \cdot 2^{-5 / 3} a^{4 / 3} t^{-1 / 3}\right) \\
& \text { (9) } \quad=c_{3} \exp \left(-3 \cdot 2^{-5 / 3} a^{4 / 3} t^{-1 / 3}\right) \text {. }
\end{aligned}
$$

Now we use (4) to obtain

(10)

$$
\begin{aligned}
\frac{16}{\pi a \sqrt{t}} & \int_{\sqrt{3} u}^{\infty} \sqrt{y} \exp \left(-a^{2} / 2 y-y^{2} / 2 t\right) d y \\
& \leq \frac{16}{\pi a} \int_{\sqrt{3} u}^{\infty} \sqrt{\frac{y}{t}} \exp \left(-y^{2} / 2 t\right) d y \\
& \leq \frac{c_{4}}{a} \sqrt{\frac{t}{\sqrt{3} u}} \exp \left(-(\sqrt{3} u)^{2} / 2 t\right) \\
& =c_{5} t^{1 / 3} a^{-4 / 3} \exp \left(-3 \cdot 2^{-5 / 3} a^{4 / 3} t^{-1 / 3}\right) .
\end{aligned}
$$

The inequality (5) gives

$$
\begin{aligned}
\frac{16}{\pi a \sqrt{t}} & \int_{0}^{2 u / 3} \sqrt{y} \exp \left(-a^{2} / 2 y-y^{2} / 2 t\right) d y \\
& \leq \frac{16}{\pi a \sqrt{t}} \int_{0}^{2 u / 3} \sqrt{y} \exp \left(-a^{2} / 2 y\right) d y \\
& \leq \frac{c_{6}}{a \sqrt{t}} 2 a^{-2}(2 u / 3)^{5 / 2} \exp \left(-a^{2} /(2(2 u / 3))\right) \\
& =c_{7} t^{1 / 3} a^{-4 / 3} \exp \left(-3 \cdot 2^{-5 / 3} a^{4 / 3} t^{-1 / 3}\right)
\end{aligned}
$$


This, (8), (9) and (10) imply that

$$
\begin{aligned}
& P\left(\sup _{0 \leq s \leq t}|Z(s)|>a\right) \\
& \quad \leq\left(c_{3}+c_{8} t^{1 / 3} a^{-4 / 3}\right) \exp \left(-3 \cdot 2^{-5 / 3} a^{4 / 3} t^{-1 / 3}\right) .
\end{aligned}
$$

Choose $c_{1}<\infty$ so large that $c_{3}+c_{8} x^{-1} \leq c_{1}$ for $x>1$ and $c_{1} \exp \left(-3 \cdot 2^{-5 / 3} x\right) \geq 1$ for $x \leq 1$. Then

$$
P\left(\sup _{0 \leq s \leq t}|Z(s)|>a\right) \leq c_{1} \exp \left(-3 \cdot 2^{-5 / 3} a^{4 / 3} t^{-1 / 3}\right) .
$$

Lemma 2. Fix some $\gamma, \beta \in(0,1)$. Then for sufficiently small $u>0$ we have

$$
\begin{gathered}
P\left(\inf _{s \in[u, 3 u]} X(s)-X(\gamma u) \geq(1-2 \beta)\left(2 / 3^{1 / 2}\right)(u \log \log (1 / u))^{1 / 2}\right) \\
\geq \log (1 / u)^{-(2 / 3)(1-\beta) /(1-\gamma)}
\end{gathered}
$$

Proof. If $y>\sqrt{t}$ then (cf. Karatzas and Shreve (1988) 2.9.22)

$$
\int_{y}^{\infty} \frac{1}{\sqrt{2 \pi t}} \exp \left(-x^{2} / 2 t\right) d x \geq \frac{1}{2 y} \sqrt{\frac{t}{2 \pi}} \exp \left(-y^{2} / 2 t\right) .
$$

Thus

$$
\begin{aligned}
& P\left(X(u)-X(\gamma u) \geq(1-\beta)\left(2 / 3^{1 / 2}\right)(u \log \log (1 / u))^{1 / 2}\right) \\
& \geq \frac{((1-\gamma) u)^{1 / 2} \exp \left(-(1-\beta)^{2}(4 / 3) u \log \log (1 / u) / 2 u(1-\gamma)\right)}{2 \sqrt{2 \pi}(1-\beta)\left(2 / 3^{1 / 2}\right)(u \log \log (1 / u))^{1 / 2}} \\
& =\frac{(1-\gamma)^{1 / 2} \exp \left(-(1-\beta)^{2}(1-\gamma)^{-1}(2 / 3) \log \log (1 / u)\right)}{4 \cdot 3^{-1 / 2} \sqrt{2 \pi}(1-\beta)(\log \log (1 / u))^{1 / 2}} \\
& =\frac{(1-\gamma)^{1 / 2} \log (1 / u)^{-(2 / 3)(1-\beta)^{2} /(1-\gamma)}}{4 \cdot 3^{-1 / 2} \sqrt{2 \pi}(1-\beta)(\log \log (1 / u))^{1 / 2}} .
\end{aligned}
$$


For small $u>0$

$$
\begin{gathered}
P\left(\inf _{s \in[u, 3 u]} X(s)-X(u) \geq-\beta\left(2 / 3^{1 / 2}\right)(u \log \log (1 / u))^{1 / 2}\right) \\
\geq P\left(\inf _{s \in[u, 3 u]} X(s)-X(u) \geq-3 \sqrt{2 u}\right) \geq 1 / 2 .
\end{gathered}
$$

The Markov property applied at $u$ yields for small $u>0$

$$
\begin{aligned}
P\left(\inf _{s \in[u, 3 u]}\right. & \left.X(s)-X(\gamma u) \geq(1-2 \beta)\left(2 / 3^{1 / 2}\right)(u \log \log (1 / u))^{1 / 2}\right) \\
& \geq \frac{1}{2} \cdot \frac{(1-\gamma)^{1 / 2} \log (1 / u)^{-(2 / 3)(1-\beta)^{2} /(1-\gamma)}}{4 \cdot 3^{-1 / 2} \sqrt{2 \pi}(1-\beta)(\log \log (1 / u))^{1 / 2}} \\
& \geq \log (1 / u)^{-(2 / 3)(1-\beta) /(1-\gamma)} \cdot \quad \square
\end{aligned}
$$

Proof of Theorem 3. First we will derive the upper bound. Let

$$
f(t) \stackrel{\mathrm{df}}{=}\left(2^{5 / 4} / 3^{3 / 4}\right) t^{1 / 4}(\log \log (1 / t))^{3 / 4}
$$

Choose some $\eta>1$. Let $\gamma=\eta^{4 / 3}$ and $a=\eta f(t)$. With this choice of $a$, Lemma 1 gives

$$
\begin{aligned}
P\left(\sup _{0 \leq s \leq t}\right. & |Z(s)|>\eta f(t))=P\left(\sup _{0 \leq s \leq t}|Z(s)|>a\right) \\
& \leq c_{1} \exp \left(-3 \cdot 2^{-5 / 3} a^{4 / 3} t^{-1 / 3}\right) \\
& =c_{1} \exp \left(-\eta^{4 / 3} \log \log (1 / t)\right) \\
& =c_{1}(\log (1 / t))^{-\gamma} .
\end{aligned}
$$


Choose an arbitrary $\alpha<1$ and note that $\eta^{2} \alpha^{-1 / 4} f(s)>$ $\eta f\left(\alpha^{k}\right)$ for $s \in\left[\alpha^{k+1}, \alpha^{k}\right]$ provided $k$ is large. We apply the last formula with $t=\alpha^{k}$ to obtain for large $k$

$$
\begin{aligned}
P\left(\sup _{\alpha^{k+1} \leq s \leq \alpha^{k}}\right. & \left.|Z(s)| / f(s)>\eta^{2} \alpha^{-1 / 4}\right) \\
& \leq P\left(\sup _{\alpha^{k+1} \leq s \leq \alpha^{k}}|Z(s)|>\eta f\left(\alpha^{k}\right)\right) \\
& \leq P\left(\sup _{0 \leq s \leq \alpha^{k}}|Z(s)|>\eta f\left(\alpha^{k}\right)\right) \\
& \leq c_{1}(k \log (1 / \alpha))^{-\gamma} .
\end{aligned}
$$

We have $\sum_{k} c_{1}(k \log (1 / \alpha))^{-\gamma}<\infty$ since $\gamma>1$. The BorelCantelli lemma implies that only a finite number of events

$$
\left\{\sup _{\alpha^{k+1} \leq s \leq \alpha^{k}}|Z(s)| / f(s)>\eta^{2} \alpha^{-1 / 4}\right\}
$$

occur. It follows that a.s.

$$
\begin{aligned}
\limsup _{t \rightarrow 0} \frac{Z(t)}{\left(2^{5 / 4} / 3^{3 / 4}\right) t^{1 / 4}(\log \log (1 / t))^{3 / 4}} & =\limsup _{t \rightarrow 0} Z(t) / f(t) \\
& \leq \eta^{2} \alpha^{-1 / 4}
\end{aligned}
$$

Since $\eta$ and $\alpha$ may be chosen arbitrarily close to 1 ,

$$
\limsup _{t \rightarrow 0} \frac{Z(t)}{\left(2^{5 / 4} / 3^{3 / 4}\right) t^{1 / 4}(\log \log (1 / t))^{3 / 4}} \leq 1 \quad \text { a.s. }
$$

Next we will prove the lower bound. 
Fix an arbitrarily small $\varepsilon_{0}>0$ and find $\varepsilon, \alpha, \beta \in\left(0, \varepsilon_{0}\right)$ which satisfy the following conditions.

(i) $\gamma \stackrel{\mathrm{df}}{=} 4 \alpha^{1 / 2} /(1-2 \varepsilon)<1-2 \varepsilon$,

(ii) $\delta \stackrel{\text { df }}{=}(1-\beta) /(1-\gamma)<1$,

(iii) $(4 \alpha)^{1 / 2} \leq \varepsilon((2 / 3)(1-\alpha))^{1 / 2} / 2$,

(iv) $\left(4 \cdot 4 \alpha^{1 / 2}\right)^{1 / 2} \leq(\varepsilon / 2)(1-2 \beta)\left(2 / 3^{1 / 2}\right)(1-2 \varepsilon)^{1 / 2}$,

(v) $\varepsilon<1 / 2$.

Let $t_{k}=\alpha^{k}$ and

$$
\begin{aligned}
u_{k} & =\left((2 / 3)\left(t_{k}-t_{k-1}\right) \log \log \left(1 /\left(t_{k}-t_{k-1}\right)\right)\right)^{1 / 2} \\
& =\left((2 / 3)(1-\alpha) t_{k} \log \log \left(1 /\left((1-\alpha) t_{k}\right)\right)\right)^{1 / 2}
\end{aligned}
$$

Note that for large $k$ we have $3(1-2 \varepsilon) u_{k+1}<4 \alpha^{1 / 2} u_{k}$. 
For large $k$ we obtain using (3) and (11)

$$
\begin{aligned}
& P\left(Y\left(t_{k}\right)-Y\left(t_{k+1}\right) \in\left[(1-\varepsilon) u_{k}, 2(1-\varepsilon) u_{k}\right]\right) \\
&=\int_{(1-\varepsilon) u_{k}}^{2(1-\varepsilon) u_{k}} \frac{1}{\sqrt{2 \pi(1-\alpha) t_{k}}} \exp \left(-x^{2} / 2(1-\alpha) t_{k}\right) d x \\
& \geq \frac{1}{2(1-\varepsilon) u_{k}} \sqrt{\frac{(1-\alpha) t_{k}}{2 \pi}} \exp \left(-\left((1-\varepsilon) u_{k}\right)^{2} / 2(1-\alpha) t_{k}\right) \\
&-\frac{1}{2(1-\varepsilon) u_{k}} \sqrt{\frac{(1-\alpha) t_{k}}{2 \pi}} \exp \left(-\left(2(1-\varepsilon) u_{k}\right)^{2} / 2(1-\alpha) t_{k}\right) \\
& \geq \frac{1}{4(1-\varepsilon) u_{k}} \sqrt{\frac{(1-\alpha) t_{k}}{2 \pi}} \exp \left(-\left((1-\varepsilon) u_{k}\right)^{2} / 2(1-\alpha) t_{k}\right) \\
&= \frac{\exp \left(-(1-\varepsilon)^{2}(1 / 3) \log \log \left(1 /\left((1-\alpha) t_{k}\right)\right)\right)}{4 \sqrt{2 \pi}(1-\varepsilon)\left((2 / 3) \log \log \left(1 /\left((1-\alpha) t_{k}\right)\right)\right)^{1 / 2}} \\
&= \frac{\left(\log \left(1 /\left((1-\alpha) t_{k}\right)\right)\right)^{-(1-\varepsilon)^{2} / 3}}{8(1-\varepsilon)\left((\pi / 3) \log \log \left(1 /\left((1-\alpha) t_{k}\right)\right)\right)^{1 / 2}} \\
& \geq c_{4}\left(\log \left(1 /\left((1-\alpha) t_{k}\right)\right)\right)^{-(1-\varepsilon) / 3} \\
&= c_{4}\left(\log \left(1 /\left((1-\alpha) \alpha^{k}\right)\right)\right)^{-(1-\varepsilon) / 3}
\end{aligned}
$$

$$
\geq c_{5} k^{-(1-\varepsilon) / 3} \text {. }
$$

Let

$$
g(u) \stackrel{\mathrm{df}}{=}(1-2 \beta)\left(2 / 3^{1 / 2}\right)((1-2 \varepsilon) u \log \log (1 /(1-2 \varepsilon) u))^{1 / 2} .
$$

Since $\gamma \stackrel{\mathrm{df}}{=} 4 \alpha^{1 / 2} /(1-2 \varepsilon)<1-2 \varepsilon$, Lemma 2 implies for 
large $k$

$$
\begin{aligned}
& P\left(\inf _{s \in\left[(1-2 \varepsilon) u_{k}, 3(1-2 \varepsilon) u_{k}\right]} X(s)-X\left(4 \alpha^{1 / 2} u_{k}\right) \geq g\left(u_{k}\right)\right) \\
& \quad \geq \log \left(1 /\left((1-2 \varepsilon) u_{k}\right)\right)^{-(2 / 3)(1-\beta) /(1-\gamma)} \\
& \quad=\log \left(1 /\left((1-2 \varepsilon)\left(\frac{2}{3}(1-\alpha) t_{k} \log \log \left(1 /\left((1-\alpha) t_{k}\right)\right)\right)^{\frac{1}{2}}\right)\right)^{\frac{-2 \delta}{3}} \\
& \quad \geq \log \left(1 /\left((1-\varepsilon)\left((2 / 3)(1-\alpha) t_{k}\right)^{1 / 2}\right)\right)^{-(2 / 3) \delta} \\
& \quad=\log \left(1 /\left((1-\varepsilon)\left((2 / 3)(1-\alpha) \alpha^{k}\right)^{1 / 2}\right)\right)^{-(2 / 3) \delta}
\end{aligned}
$$

$$
\geq c_{6} k^{-(2 / 3) \delta} \text {. }
$$

Let

$$
\begin{aligned}
A_{k} \stackrel{\text { df }}{=}\left\{\inf _{s \in\left[(1-2 \varepsilon) u_{k}, 3(1-2 \varepsilon) u_{k}\right]} X(s)-X\left(4 \alpha^{1 / 2} u_{k}\right) \geq g\left(u_{k}\right)\right\} \\
\cap\left\{Y\left(t_{k}\right)-Y\left(t_{k+1}\right) \in\left[(1-\varepsilon) u_{k}, 2(1-\varepsilon) u_{k}\right]\right\} .
\end{aligned}
$$

By (13), (14) and the independence of $X$ and $Y$,

$$
P\left(A_{k}\right) \geq c_{5} k^{-(1-\varepsilon) / 3} c_{6} k^{-(2 / 3) \delta} .
$$

Since $-(1-\varepsilon) / 3-(2 / 3) \delta>-1$,

$$
\sum_{k} c_{5} k^{-(1-\varepsilon) / 3} c_{6} k^{-(2 / 3) \delta}=\infty
$$

Recall that $3(1-2 \varepsilon) u_{k+1}<4 \alpha^{1 / 2} u_{k}$ for large $k$. It follows that for some large $k_{0}$, the events $\left\{A_{k}\right\}_{k \geq k_{0}}$ are independent. 
Thus the Borel-Cantelli Lemma implies that with probability 1 infinitely many events $A_{k}$ occur.

We have chosen $\alpha$ and $\varepsilon$ so that

$$
(4 \alpha)^{1 / 2} \leq \varepsilon((2 / 3)(1-\alpha))^{1 / 2} / 2
$$

The standard LIL implies that with probability 1 for all sufficiently large $k$ we have

$$
\begin{aligned}
\left|Y\left(t_{k+1}\right)\right| & \leq\left(4 t_{k+1} \log \log \left(1 / t_{k+1}\right)\right)^{1 / 2} \\
& =\left(4 \alpha t_{k} \log \log \left(1 / \alpha t_{k}\right)\right)^{1 / 2} \\
& \leq \varepsilon\left((2 / 3)(1-\alpha) t_{k} \log \log \left(1 /\left((1-\alpha) t_{k}\right)\right)\right)^{1 / 2} \\
& =\varepsilon u_{k} .
\end{aligned}
$$

We have assumed that

$$
\left(4 \cdot 4 \alpha^{1 / 2}\right)^{1 / 2} \leq(\varepsilon / 2)(1-2 \beta)\left(2 / 3^{1 / 2}\right)(1-2 \varepsilon)^{1 / 2} .
$$

The LIL for the Brownian motion $X$ shows that for large $k$

$$
\begin{aligned}
& \left|X\left(4 \alpha^{1 / 2} u_{k}\right)\right| \\
& \leq\left(4 \cdot 4 \alpha^{1 / 2} u_{k} \log \log \left(1 /\left(4 \alpha^{1 / 2} u_{k}\right)\right)\right)^{1 / 2} \\
& \leq(\varepsilon / 2)(1-2 \beta)\left(2 / 3^{1 / 2}\right)\left((1-2 \varepsilon) u_{k} \log \log \left(1 /\left(4 \alpha^{1 / 2} u_{k}\right)\right)\right)^{1 / 2} \\
& \leq \varepsilon(1-2 \beta)\left(2 / 3^{1 / 2}\right)\left((1-2 \varepsilon) u_{k} \log \log \left(1 /(1-2 \varepsilon) u_{k}\right)\right)^{1 / 2} \\
& (16) \\
& \quad=\varepsilon g\left(u_{k}\right) .
\end{aligned}
$$


Recall that infinitely many events

$$
\begin{aligned}
A_{k}= & \left\{\inf _{s \in\left[(1-2 \varepsilon) u_{k}, 3(1-2 \varepsilon) u_{k}\right]} X(s)-X\left(4 \alpha^{1 / 2} u_{k}\right) \geq g\left(u_{k}\right)\right\} \\
& \cap\left\{Y\left(t_{k}\right)-Y\left(t_{k+1}\right) \in\left[(1-\varepsilon) u_{k}, 2(1-\varepsilon) u_{k}\right]\right\}
\end{aligned}
$$

occur a.s. By (15) and (16), the events $\left\{\left|Y\left(t_{k+1}\right)\right| \leq \varepsilon u_{k}\right\}$ and $\left\{\left|X\left(4 \alpha^{1 / 2} u_{k}\right)\right| \leq \varepsilon g\left(u_{k}\right)\right\}$ hold for all large $k$ a.s. Hence

$$
Y\left(t_{k}\right) \in\left[(1-2 \varepsilon) u_{k}, 3(1-2 \varepsilon) u_{k}\right]
$$

and

$$
\inf _{s \in\left[(1-2 \varepsilon) u_{k}, 3(1-2 \varepsilon) u_{k}\right]} X(s) \geq(1-\varepsilon) g\left(u_{k}\right)
$$

for infinitely many $k$ a.s. It follows that $Z\left(t_{k}\right) \geq(1-\varepsilon) g\left(u_{k}\right)$ occurs i.o. with probability 1 . It is easy to check that

$$
\frac{(1-2 \beta)\left(2 / 3^{1 / 2}\right)\left((1-2 \varepsilon) u_{k} \log \log \left(1 /(1-2 \varepsilon) u_{k}\right)\right)^{1 / 2}}{(1-2 \beta)\left(2 / 3^{1 / 2}\right)\left((1-2 \varepsilon)\left((2 / 3)(1-\alpha) t_{k}\right)^{1 / 4}\left(\log \log \left(1 / t_{k}\right)\right)^{3 / 4}\right.}
$$

goes to 1 as $k \rightarrow \infty$. Hence

$$
\begin{aligned}
& \limsup _{k \rightarrow \infty} \frac{Z\left(t_{k}\right)}{\left(2^{5 / 4} / 3^{3 / 4}\right) t_{k}^{1 / 4}\left(\log \log \left(1 / t_{k}\right)\right)^{3 / 4}} \times \\
& \quad \times \frac{1}{(1-2 \beta)(1-2 \varepsilon)^{1 / 4}(1-\alpha)^{1 / 4}} \\
& =\limsup _{k \rightarrow \infty} \frac{Z\left(t_{k}\right)}{\frac{2(1-2 \beta)}{\sqrt{3}}\left((1-2 \varepsilon)\left(\frac{2}{3}(1-\alpha) t_{k}\right)^{1 / 4}\left(\log \log \left(1 / t_{k}\right)\right)^{3 / 4}\right.} \\
& =\limsup _{k \rightarrow \infty} \frac{Z\left(t_{k}\right)}{(1-2 \beta) \frac{2}{\sqrt{3}}\left((1-2 \varepsilon) u_{k} \log \log \left(1 /(1-2 \varepsilon) u_{k}\right)\right)^{1 / 2}} \\
& =\limsup _{k \rightarrow \infty} \frac{Z\left(t_{k}\right)}{g\left(u_{k}\right)} \\
& \geq(1-\varepsilon) \text { a.s. }
\end{aligned}
$$


Since $\varepsilon, \alpha$ and $\beta$ may be chosen arbitrarily close to 0 , we obtain

$$
\limsup _{t \rightarrow 0} \frac{Z(t)}{\left(2^{5 / 4} / 3^{3 / 4}\right) t^{1 / 4}(\log \log (1 / t))^{3 / 4}} \geq 1 \quad \text { a.s. }
$$

This and (12) prove Theorem 3.

\section{REFERENCES}

1. T. Funaki, Probabilistic construction of the solution of some higher order parabolic differential equations, Proc. Japan Acad. 55 (1979), 176-179.

2. K.J. Hochberg, A signed measure on path space related to Wiener measure, Ann. Probab. 6 (1978), 433-458.

3. I. Karatzas and S. Shreve, Brownian Motion and Stochastic Calculus, Springer, New York, 1988.

4. V. Yu. Krylov, Some properties of the distribution corresponding to the equation $\partial u / \partial t=(-1)^{q+1} \partial^{2 q} u / \partial x^{2 q}$, Soviet Math. Dokl. 1 (1960), 760-763.

5. A. Mądrecki and M. Rybaczuk, New Feynman-Kac type formula, (preprint) (1992).

6. W. Vervaat, Sample path properties of self-similar processes with stationary increments, Ann. Probab. 13 (1985), 1-27.

Department of Mathematics, GN-50 Seattle, WA 98195 